\title{
Occupational mycoses
}

\author{
SHI ZHICHENG ${ }^{1}$ AND LEI PANGCHENG ${ }^{2}$ \\ From the Departments of Occupational Medicine, ${ }^{1}$ and Dermatology, ${ }^{2}$ Third Hospital, Beijing Medical \\ University, Beijing, China
}

Moulds exist widely in nature and because of their ability to catabolise or synthesise certain complex organic substances some are used in industries such as food, brewing, and drug manufacture as raw material or catalysts. In addition, some pathogenic moulds may live and reproduce in fur, straws, wood, grain, and dusts. In recent years there has been a real increase in the number of patients suffering from disease caused by mycobacteria. ${ }^{1} \mathrm{~A}$ high proportion of these patients work in dusty occupations or other trades. $^{23}$ We report here on some cases of occupational mycoses.

Group 1-Four animal handlers who fed rabbits suffered from Tinea corporis on the face and hands. They were infected within two months of beginning work. Trichophyton gypseum was found in cultures both from the rabbits' fur and the desquamated epidermis of the feeders' skin lesions.

Group 2-Nine silkreeling workers were exposed to the spores of Beauveria vuillemin. Two weeks later, six had developed contact and allergic dermatitis. Beauveria vuillemin was seen in cultures of the skin crust in three patients; eosinophilia was present in two cases.

Group 3-A carpenter aged 59 noted verrucous nodules growing on his left leg that spread slowly upward with the growth of satellite lesions. The nodules were bright, dark red, and full of pus and the skin around the affected areas was oedematous. As the lesions developed the leg became swollen and covered with many nodules with plaque like and cicatrical lesions. A skin biopsy showed Chromoblasto mycosis and Dematiacious mould was found on culture. Oral potassium iodide, amphotericin B injected locally, and freezing with liquid nitrogen were ineffective and the left leg had finally to be amputated.

Group 4-Nine farmers had disease of the eyes during a busy harvest, four suffering from acute keratitis and kerato ulcer and five from acute conjunctivitis. Fusarium link exFr was found on cultures of the conjunctival sac.

Group 5-A dynamite worker worked in a small

Accepted 23 September 1985 and dark room, the walls and floor of which were damp and wet. To make matters worse the raw materials-chaff and firewood-were also wet and mouldy. Two months before his admission he had suffered from pneumonia that was resistant to antibiotics. On admission, the $x$ ray film showed irregular and heavy lung markings, especially in the lower zones. Small hazy, patchy shadows with a tendency to conglomerate were present. Aspergillus link, Penicillium link exFr, and Candida albicans (Robin) were found several times both in the patient's sputum and in the soil from the wall and floor of the workshop. The symptoms and the $x$ ray changes improved remarkably after antimycotic treatment. His final diagnosis was mycotic interstitial pneumonia with mild fibrosis.

Group 6-Five blanket makers washed and cleaned fur for more than 10 years. The workshop was dusty. They all suffered from chronic asthmatic bronchitis complicated with mycotic infection. Cultures of sputum and dust showed Penicillium link and $C$ albicans (Robin).

Group 7-A woman of 34 entered an ill ventilated warehouse to remove mouldy grain and rice. She worked in the warehouse for about half an hour. Soon afterwards she became short of breath and noted itching in her throat, palpitations, and nausea. Four hours later she had chills, fever $\left(38.6^{\circ} \mathrm{C}\right)$, and wheezing; a chest $x$ ray film result was negative. The clinical diagnosis was acute allergic asthma. Mycotic spores were cultured from the mouldy grain.

Group 8-In a workshop producing ATP by the phosphorylate method 12 workers were exposed to Penicillium citrinum. After finishing one day's work all of them complained of chills, fever, weakness, lassitude, headache, tightness of the chest, dyspnoea, cough and expectoration. These symptoms subsided within six to 24 hours. $X$ ray examination showed heavy lung markings in three cases and sputum culture showed Penicillium citrinum in two. Cellulose acetate strip electrophoresis showed a rise of $\gamma$-globulin in eight cases; serum IgG and IgA levels were increased in nine and IgM levels in three. The precipitin reaction was positive in eight cases and the 
clinical diagnosis was allergic reaction due to the inhalation of Penicillium citrinum.

Group 9-In a workshop producing complex esterases roots of malt were used as the raw material. After working in a pulverisation process all 12 workers developed a low fever which lasted for 10 to 20 hours with malaise, joint pains, conjunctivitis, dryness in the throat, tightness in the chest, cough with expectoration, and loss of appetite. These symptoms disappeared within one to four days after stopping work. Physical and $x$ ray examination results were normal but serum $\gamma$-globulin levels were increased in 11 workers and two had eosinophilia. Rhizopus ehrenb ex corda was found in cultures of the raw material, the pulverised powder, throat swabs, and sputum. The clinical diagnosis was systemic allergic reaction due to the inhalation of mycotic spores.
According to the clinical manifestations and the pathogenesis mentioned above the cases may be divided into two types: type 1 in which there is direct infection by moulds that invade the skin (groups 1, 2 , and 3), the eyes (group 4), or the respiratory tract (groups 5 and 6) and type 2 in which there is an allergic reaction (groups 7,8 , and 9). With the rapid development of industry and agriculture more attention will need to be given to occupational mycoses.

\section{References}

${ }^{1}$ Editorial. Opportunist mycobacteria. Lancet 1981;1:424.

${ }^{2}$ Marks J. Occupation and kansasii infection in Cardiff residents. Tubercle 1975;56:311-3.

${ }^{3}$ British Thoracic and Tuberculosis Association. Opportunist mycobacterial pulmonary infection and occupational dust exposure: an investigation in England and Wales. Tubercle 1975;56:295. 